\title{
Binge drinking: um estudo bibliométrico (1999-2010) dos artigos publicados na base de dados SciELO
}

\author{
Binge drinking: a bibliometric study \\ (1999-2010) of articles published \\ in the ScIELO Database
}

Luis Fernando Resende ARANTES

\section{Resumo}

O objetivo deste estudo foi realizar uma pesquisa bibliométrica dos artigos publicados na base de dados Scientific Electronic Library Online, a fim de examinar os avanços científicos em relação ao comportamento de beber em excesso, nas diversas populações identificadas. Utilizaram-se os descritores binge e drinking, em inglês, na referida base de dados, tendo sido encontrados 22 trabalhos, sete dos quais repetidos pelo sistema de busca, resultando em 15 artigos distintos publicados entre 1999 e 2010. Foram criadas oito categorias de análise. Verificou-se a predominância de: parcerias entre universidades e centros de pesquisa (66,7\%); pesquisas multiprofissionais (66,7\%); publicações em revistas brasileiras (73,3\%); uso do idioma inglês (53,3\%); emprego do método quantitativo (86,7\%); população adulta (60,0\%); fatores associados ao binge drinking (26,7\%); e definições quantitativas do comportamento analisado (100,0\%). Os resultados apontam a baixa produção brasileira a respeito do tema, bem como a necessidade de pesquisas qualitativas a fim de compreender melhor a complexidade do binge drinking.

Unitermos: Alcoolismo. Bases de dados. Bebida alcoólica. Pesquisa bibliométrica.

\begin{abstract}
The aim of this research was to undertake a bibliometric study of articles published in the Scientific Electronic Library Online database in order to examine scientific advances regarding binge-drinking behaviour in the different populations identified. We searched forkey words "binge" and "drinking" in journals and found 22 papers in the SciELO database. Seven of these had been repeated by the search system, therefore resulting in 15 separate articles dating from 1999-2010. We then created eight categories for analysis. We encountered a predominance of; partnerships between universities and research centers (66.7\%); multidisciplinary research (66.7\%); publications in Brazilian journals (73.3\%); articles in English (53.3\%); use of quantitative methods (86.7\%); adults (60\%); factors associated with binge drinking (26.7\%); and quantitative definitions of binge drinking (100\%). The results point to the low number of articles produced in Brazil on the subject and the need for qualitative research to better understand the complexity of the phenomenon.
\end{abstract}

Uniterms: Alcoholism. Databases. Drinking. Bibliometric research.

- Universidade de Brasília, Programa de Pós-Graduação em Psicologia Clínica e Cultura. Campus Universitário Darci Ribeiro, 70910-900, Brasília, DF, Brasil. E-mail:<lufearantes@gmail.com>. 
Desde tempos imemoriais o uso de drogas acompanha as distintas configurações sociais humanas, seja em relação ao aspecto qualitativo do uso, seja em relação à quantidade consumida pelos usuários. $\mathrm{O}$ álcool, droga mais consumida do mundo, frequentemente apresenta graves consequências físicas, sociais e psíquicas para aqueles que a utilizam.

Beber consumindo um volume excessivo de álcool num curto espaço de tempo é definido na literatura internacional como binge drinking ou "beber em binge" (Laranjeira, Pinsky, Zalesky \& Caetano, 2007). Wechsler e Nelson (2001) introduziram a definição de binge drinking como o consumo de cinco ou mais doses num período de pelo menos uma vez nas últimas duas semanas para homens, e quatro ou mais doses no período para mulheres. Uma dose alcoólica é equivalente a uma lata de $350 \mathrm{~mL}$ de cerveja, um pequeno copo (120-150mL) de vinho ou uma dose (30mL-50mL) de destilado (cachaça, whisky e outros) (Prais, Loyola Filho, Firmo, Lima-Costa \& Uchoa, 2008).

O presente estudo realizou uma pesquisa bibliométrica dos artigos publicados na base de dados Scientific Electronic Library Online (SciELO), buscando examinar os avanços científicos em relação ao comportamento de beber em excesso ou binge drinking, nas diversas populações identificadas. Procurou-se analisar as publicações realizadas sobre o tema nos últimos 11 anos, entre 1999 e 2010.

O padrão de consumo denominado binge drinking é visto como um comportamento que intensifica os danos relacionados ao álcool (Wechsler \& Nelson, 2001), apresentando riscos à saúde e consequências sociais não só ao bebedor quanto àqueles que estão próximos a ele (C.M. Silveira, C.C. Silveira, Silva, A.G.A. Silveira \& Andrade, 2008).

Simões, Matos e Batista-Foguet (2006) apontam que os comportamentos de consumo abusivo trazem consequências graves a curto, médio e longo prazo, em várias esferas da vida dos usuários, nomeadamente pessoal, interpessoal, familiar, escolar e profissional.

Naimi et al. (2003) ressaltam ainda que o consumo abusivo de álcool (binge drinking) está associado a importantes consequências físicas, psicológicas e sociais, tais como: acidentes automobilísticos, suicídio, 254 pressão arterial elevada, infarto do miocárdio, gastrite, pancreatite, dificuldades no controle do diabetes e violência interpessoal.

No estudo de Vieira, Ribeiro, Romano e Laranjeira (2007), identificou-se que os adolescentes pesquisados relataram ter passado mal por ter bebido (17,9\%), arrependimento por algo que fizeram sob o efeito do álcool (11,0\%), blackout (9,8\%) e ter brigado após beber $(5,0 \%)$. Mais da metade $(55,0 \%)$ dos estudantes conhecia alguém que sofreu acidente de trânsito provocado por motorista embriagado. Os prejuízos citados estão relacionados principalmente ao abuso agudo, e as complicações mais relatadas foram concordantes com as propriedades farmacológicas do álcool sobre o organismo, principalmente ressaca $(18,4 \%)$.

As expectativas de efeitos sobre o comportamento abusivo de álcool estão, em grande parte, próximas à fase dos efeitos estimulantes da substância (Araújo \& Gomes, 1998). No estudo os participantes relataram que, além de produzir desinibição e alegria, o álcool facilita comportamentos sociais, influi sobre o raciocínio, libera a sexualidade e ajuda a esquecer problemas. 0 álcool aparece como indispensável, como sendo aquilo que dá graça à festa, que faz com que todos fiquem amigos e entrem em harmonia com o ambiente.

Independentemente da quantidade consumida habitualmente, o binge drinking ou beber em excesso é um indicador-chave de abuso de álcool (Naimi et al., 2003). O risco de dependência advindo desses comportamentos abusivos está na incapacidade de o abusador enfrentar um grande número de situações sem o álcool, pois este passa a ser visto como o verdadeiro agente, responsável pelo bom desempenho e culpado pelas falhas (Araújo \& Gomes, 1998). Silva et al. (2007) relataram a extrema importância dos programas que visam ao controle do uso de álcool, principalmente, entre jovens, para a melhoria do bem-estar psicológico.

\section{Método}

\section{Procedimentos}

Para a pesquisa das publicações sobre o comportamento de beber em excesso, utilizaram-se os descritores binge e drinking, em inglês, a fim de analisar as publicações na base de dados SciELO. A pesquisa compreendeu o período dos últimos 11 anos (1999-2010), 
tendo sido realizada em maio de 2010, com atualizações em junho do mesmo ano.

Inicialmente, fez-se uma leitura breve do material, sobretudo dos resumos publicados, a fim de identificar o assunto discutido. A partir dos descritores foram encontrados 22 trabalhos publicados na base de dados SciELO, porém 7 dos trabalhos identificados pelo sistema de busca eram repetidos, resultando no número final de 15 artigos distintos.

As categorias e subcategorias analisadas no presente estudo foram:

1) $O$ país da universidade ou centro onde foi realizado o estudo: parceria de universidades (Brasil e país estrangeiro), parceria de centros e universidades brasileiros, parceria de centros e universidades estrangeiros, universidade brasileira, órgão público;

2) A área do profissional que publicou o artigo: multiprofissional, Medicina, Psicologia;

3) O país da revista em que o artigo foi publicado: revista brasileira, revista norte-americana, revista espanhola;

4) O idioma em que foi publicado: inglês, português, espanhol;

5) $\bigcirc$ método utilizado na investigação: estudo quantitativo, estudo qualitativo, revisão de literatura/ meta-análise, editorial ou comentários;

6) A população que fez parte do estudo: adultos; adolescentes; idosos; adultos e adolescentes;

7) Os assuntos tratados na investigação: fatores associados ao excesso de bebida, padrão de consumo, percepção de saúde, direção sob efeito de álcool, consumo episódico excessivo;

8) A definição do comportamento binge drinking: definição quantitativa (número de doses em determinado período), definição qualitativa.

Cada artigo analisado foi classificado em apenas uma subcategoria dentro das categorias apresentadas.

\section{Resultados}

O primeiro ponto a ser destacado é o número de publicações na base de dados SciELO. O quantitativo de artigos encontrados apresenta oscilações ao longo do período proposto, segundo os dados analisados a partir dos descritores binge e drinking,

Na Figura 1 identifica-se o número total de publicações na SciELO entre 1999 e 2010. Nenhum artigo foi encontrado na referida base nos anos de 2001, 2002, 2003, 2006 e 2010. Em 2008 encontra-se o volume mais expressivo de publicações sobre a temática, ano em que foram encontrados sete artigos. Nos demais anos, o quantitativo varia entre um e dois trabalhos publicados.

A maioria dos artigos publicados na base foi realizada por meio de parcerias entre universidades e centros, sejam universidades brasileiras e estrangeiras, sejam ainda universidades e centros brasileiros, com $26,7 \%$ cada qual. Ao todo, as parcerias compreenderam $66,7 \%$ da amostra.

Na análise verifica-se também a predominância dos estudos realizados por meio de grupos de áreas distintas, como Medicina, Psicologia, Nutrição e Educação Física, dentre outras, caracterizando a maioria dos trabalhos (66,7\%) como multiprofissionais.

Ainda que as revistas brasileiras sejam maioria na base de dados analisada (73,3\%), o idioma inglês esteve presente em 53,3\% dos trabalhos.

Quanto ao método de pesquisa, verifica-se que $86,7 \%$ dos trabalhos foram desenvolvidos a partir de abordagens quantitativas, sobretudo por meio da aplicação de questionários e instrumentos objetivos. 0 percentual restante refere-se a publicações que utilizaram o método de revisão de literatura ou meta-análise.

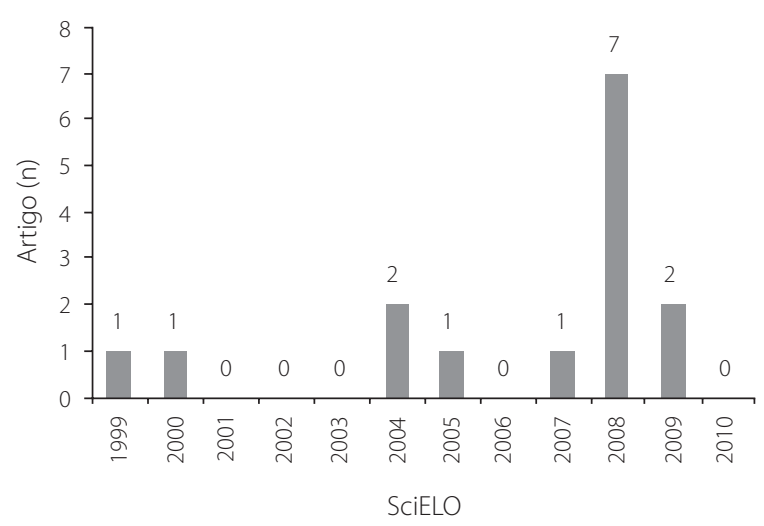

Figura 1. Número de artigos publicados na base SciELO entre 1999 e 2010.

Nota: SciELO: Scientific Electronic Library Online. 
Tabela 1. Assunto tratado na investigação.

\begin{tabular}{lcc}
\hline Assuntos publicados & Frequência & Percentual \\
\hline Fatores associados ao beber pesado & 4 & 26,70 \\
Padrões de consumo & 3 & 20,00 \\
Percepção de saúde & 2 & 13,30 \\
Consumo episódico excessivo & 1 & 6,70 \\
Direção sob efeito de álcool & 1 & 6,70 \\
Outros & 4 & 26,70 \\
\hline Total & 15 & 100,00 \\
\hline
\end{tabular}

A população adulta foi a mais pesquisada na amostra deste estudo (60,0\%), seguida pelos adolescentes e idosos, cada qual com 13,3\%. Os fatores associados ao beber pesado ou binge drinking foi o assunto predominante nas publicações da base SciELO, seguido pelos padrões de consumo das populações analisadas (Tabela 1).

O comportamento de beber pesado ou binge drinking foi definido por todos os artigos analisados por meio do número de doses consumidas em determinada ocasião: 5 ou mais doses para homens e 4 ou mais doses para mulheres. Nenhuma das definições destacou variáveis subjetivas dos participantes, por meio de uma abordagem qualitativa.

\section{Discussão}

Composta principalmente por revistas brasileiras, o baixo número de artigos publicados na base de dados SciELO é um indicador da baixa produção brasileira a respeito da temática de abuso de álcool, sobretudo o comportamento de beber em excesso ou binge drinking. Uma das possíveis causas para esse resultado é a escassez de investimentos e recursos públicos e privados para o desenvolvimento de pesquisas calcadas na realidade brasileira.

Na base de dados analisada existe uma tendência de trabalhos em parceria de universidades brasileiras e estrangeiras, ou ainda entre universidades e centros brasileiros. As pesquisas realizadas em diferentes locais permitem o acesso a maior quantidade de participantes e a possibilidade de análises estatísticas comparativas, segundo as diferenças regionais, nacionais ou internacionais.

A complexidade do tema relativo ao consumo 256 abusivo de álcool, sobretudo as consequências desse comportamento tanto para os abusadores quanto para as demais pessoas, sugere que as intervenções ou as elaborações teóricas sejam pautadas na perspectiva multiprofissional, conforme encontrado em 66,7\% da amostra.

Enquanto meio de comunicação científica internacional, o idioma inglês esteve presente em mais da metade dos trabalhos (53,3\%), ainda que a maioria das revistas presentes na base SciELO fossem brasileiras. Esse dado aponta a perspectiva de internacionalização das revistas nacionais e a possibilidade de publicações em língua estrangeira.

A fim de obter resultados com amostras significativas da população, grande parte dos estudos (86,7\%) utilizou instrumentos quantitativos para obtenção dos dados sobre o comportamento abusivo de álcool. 0 percentual restante refere-se aos trabalhos de revisão de literatura ou meta-análise. O método qualitativo de coleta de dados não se mostrou presente na amostra, ainda que fatores psicológicos, físicos e sociais estejam presentes no comportamento abusivo de álcool.

A população adulta, com idade superior a 18 anos, foi o foco de mais da metade dos estudos analisados (60\%), ainda que na maioria dos casos o primeiro contato com a bebida alcoólica se dê na fase da adolescência, entre 12 e 18 anos. Fatores associados ao binge drinking, tais como risco cardiovascular, condutas de risco, aumento da mortalidade e comportamento agressivo foram o assunto predominante na amostra.

As definições quantitativas do binge drinking, pautadas na quantidade de doses alcoólicas ingeridas em determinada ocasião foram unanimidade nas pesquisas analisadas. Esse resultado, aliado à complexidade do comportamento abusivo de álcool, sugere que pesquisas qualitativas sejam desenvolvidas a fim de compreender melhor os diferentes fatores associados ao binge drinking, ressaltando variáveis subjetivas que influenciam o comportamento do abusador.

O presente estudo apresenta algumas limitações que devem ser discutidas. A amostra utilizada como método de pesquisa mostra-se pequena diante do volume de publicações sobre o tema nos periódicos da Coordenação de Aperfeiçoamento de Pessoal de Nível Superior (CAPES). A revisão bibliométrica pode ser expandida para outras bases de dados, a fim de obter resultados estatísticos mais significativos. 
Outra questão relevante é o possível viés das publicações de profissionais vinculados a universidades ou centros de pesquisa. Aqueles que lidam com intervenções práticas diárias, sejam médicos, psicólogos ou outros profissionais, e que não possuem vínculo com essas instituições, possivelmente são os que publiquem em menor frequência, se comparados aos demais. Diante da possibilidade de que muitos trabalhos práticos não estariam contemplados nas publicações científicas, tal viés de publicação dificultaria a generalização dos estudos de revisão de literatura.

Apesar das limitações discutidas, o presente trabalho contribui para o mapeamento das publicações na base SciELO, em que predominam revistas brasileiras, e direciona para futuras investigações sobre o comportamento de beber em excesso ou binge drinking.

\section{Referências}

Araújo, L. B., \& Gomes, W. B. (1998). Adolescência e as expectativas em relação aos efeitos do álcool. Psicologia: Reflexão e Crítica, 11 (1), 5-33.

Laranjeira, R., Pinsky, I., Zalesky, M., \& Caetano, R. (2007). I levantamento nacional sobrepadrões do consumo deálcool na população brasileira. Brasília: Secretaria Nacional Antidrogas.
Naimi, T. S., Brewer, R. D., Mokdad A., Denny C., Serdula, M. K., \& Marks, J. S. (2003). Binge drinking among US adults. JAMA, 289 (1), 70-75.

Prais, H. A. C., Loyola Filho, A. I., Firmo, J. O. A., Lima-Costa, M. F., \& Uchoa, E. (2008). A population-based study on binge drinking among elderly Brazilian men: evidence from the Belo Horizonte and Bambuí health surveys. Revista Brasileira de Psiquiatria, 30 (2), 118-123.

Silva, R. A., Horta, B. L., Pontes, L. M., Faria, A. D., Souza, L. D. M., \& Cruzeiro, A. L. S. (2007). Bem-estar psicológico e adolescência:fatores associados. Cadernos de SaúdePública, $23(5), 1113-1118$.

Silveira, C. M., Silveira, C. C., Silva, J. G., Silveira, A. G. A., \& Andrade, L. H. G. (2008). Epidemiologia do beber pesado e beber pesado episódico no Brasil: uma revisão sistemática da literatura. Revista de Psiquiatria Clínica, 35 (1), 31-38.

Simões, C., Matos, M. G., \& Batista-Foguet, J. (2006). Consumo de substâncias na adolescência: um modelo explicativo. Psicologia, Saúde \& Doenças, 7 (2), 147-164.

Vieira, D. L., Ribeiro, M., Romano, M., \& Laranjeira, R. R. (2007). Álcool e adolescentes: estudo para implementar políticas municipais. Revista de Saúde Pública, 41 (3), 396-403.

Wechsler, H., \& Nelson, T. F. (2001). Binge drinking and the American college student: what's five drinks? Review Psychology of Addictive Behavior, 15 (4), 287-291.

Recebido em: 3/8/2010

Aprovado em: 27/9/2011 
